\title{
Particle Loss Calculator - a new software tool for the assessment of the performance of aerosol inlet systems
}

\author{
S.-L. von der Weiden ${ }^{1,2}$, F. Drewnick ${ }^{1}$, and S. Borrmann ${ }^{1,2}$ \\ ${ }^{1}$ Particle Chemistry Department, Max Planck Institute for Chemistry, Joh.-J.-Becher-Weg 27, 55128 Mainz, Germany \\ ${ }^{2}$ Institute for Atmospheric Physics, Johannes Gutenberg University, Joh.-J.-Becher-Weg 21, 55128 Mainz, Germany
}

Received: 25 March 2009 - Published in Atmos. Meas. Tech. Discuss.: 22 April 2009

Revised: 7 August 2009 - Accepted: 19 August 2009 - Published: 3 September 2009

\begin{abstract}
Most aerosol measurements require an inlet system to transport aerosols from a select sampling location to a suitable measurement device through some length of tubing. Such inlet systems must be optimized to minimize aerosol sampling artifacts and maximize sampling efficiency. In this study we introduce a new multifunctional software tool (Particle Loss Calculator, PLC) that can be used to quickly determine aerosol sampling efficiency and particle transport losses due to passage through arbitrary tubing systems. The software employs relevant empirical and theoretical relationships found in established literature and accounts for the most important sampling and transport effects that might be encountered during deployment of typical, ground-based ambient aerosol measurements through a constant-diameter sampling probe. The software treats non-isoaxial and nonisokinetic aerosol sampling, aerosol diffusion and sedimentation as well as turbulent inertial deposition and inertial deposition in bends and contractions of tubing. This software was validated through comparison with experimentally determined particle losses for several tubing systems bent to create various diffusion, sedimentation and inertial deposition properties. As long as the tube geometries are not "too extreme", agreement is satisfactory. We discuss the conclusions of these experiments, the limitations of the software and present three examples of the use of the Particle Loss Calculator in the field.
\end{abstract}

\section{Introduction}

Aerosols affect the climate on a global scale (IPCC, 2007) as well as impact human and animal health on a local scale

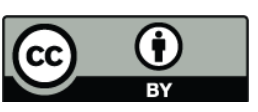

Correspondence to:

S.-L. von der Weiden

(sl.vonderweiden@mpic.de)
(Brunekreef and Holgate, 2002). The great influence of aerosols over such a wide range of scales puts measurements of integral, physical, and chemical aerosol properties such as size-distributions and size-resolved aerosol composition in high demand. Atmospheric aerosol particles cover a size range of more than four orders of magnitude, from freshly nucleated clusters having aerodynamic sizes of a few nanometers, to aged and cumulated particles and crustal dust particles with sizes of several micrometers, to cloud droplets with sizes on the order of millimeters. Aerosols are comprised of a large variety of materials having diverse properties that change, along with overall aerosol concentration, over time (McMurry, 2000). These characteristics place high demands on measurement systems and instrumentation used to investigate them.

In recent years, both universities and research institutes have been developing and improving aerosol measurement instrumentation (e.g., Winklmayr et al., 1991; Gard et al., 1997; Weber et al., 2001; Orsini et al., 2003; Drewnick et al., 2005; Sagharfifar et al., 2009) to enable measurement of a variety of aerosol properties with high temporal resolution and accuracy. One important trend in aerosol science is the development of mobile laboratories which are able to measure aerosol properties while underway. These mobile laboratories are typically equipped with instrumentation having high temporal resolutions and measure aerosol particle properties (and also gas loadings) in real time (Bukowiecki, 2002; Kittelson et al., 2000; Kolb et al., 2004; Pirjola et al., 2004).

Because of this, high demands are placed on the sampling and inlet system that transports aerosols from outside of the vehicle to each measurement device inside. Ideally, the inlet system performs its function without changing aerosol characteristics, composition, concentration, or size distribution. In reality, sampling is non-ideal (non-isoaxial and/or nonisokinetic) and transport losses due to a number of mechanisms (e.g. diffusion, sedimentation, inertial deposition)

Published by Copernicus Publications on behalf of the European Geosciences Union. 
are often not negligible. These effects can cause significant changes in the aerosol properties prior to measurement giving rise to unquantifiable uncertainties in spite of the fact that carefully calibrated measurement instrumentation is used. For instance, operational loss mechanisms during sampling and transport depend on particle size. Small particles with an aerodynamic size below about $100 \mathrm{~nm}$ and large particles with a size above about $0.5 \mu \mathrm{m}$ are particularly affected. When not accounted for, such non-uniform particle losses can alter size-distribution measurements making a bimodal size distribution appear monomodal. Such discrimination also affects chemical composition measurements since the substances comprising the particles are not equally distributed with respect to particle size (Appel et al., 1988; Huebert et al., 1990; McMurry, 2000). To avoid erroneous measurement results, new inlet systems should be optimized prior to construction and properties of existing inlet systems should be characterized to enable correction of measurements.

Recently, the Max Planck Institute for Chemistry in Mainz developed a mobile laboratory ("MoLa") for flexible and mobile measurements of aerosol and gas concentration and composition. As part of this development, the software Particle Loss Calculator (PLC) was conceived as an efficient, flexible method for calculating particle losses due to sampling to aid in inlet design for MoLa. The current version of the Particle Loss Calculator can be downloaded on http://www.mpch-mainz.mpg.de/ drewnick/PLC. The PLC is written in the scientific programming environment IGOR Pro, which is also necessary to run the software. A free IGOR trial version is available on www.wavemetrics.com.

In Sect. 2, relevant aerosol loss mechanisms treated by the Particle Loss Calculator are described. The basis for selection and use of the equations implemented for each loss calculation is described in Sect. 3. In Sect. 4, comparison between validation experiments and calculations performed using the Particle Loss Calculator are discussed while three sample applications of this calculator are described in Sect. 5 .

\section{Particle loss mechanisms}

An aerosol sampling system generally consists of a sampling probe and transport lines and, depending on aerodynamic particle size, a variety of particle loss mechanisms could be operative in any given system (e.g., Levin, 1957; Davies, 1966; Fuchs, 1975; Vincent, 1989; Willeke and Baron, 2005). In the sampling probe, non-representative sampling comes from non-isoaxial and non-isokinetic conditions related to movement of the air entering the probe. Here, such losses due to extraction of aerosol particles from ambient air into the sampling system are described using the "sampling efficiency" (Sect. 2.3). The main particle loss mechanisms operative during transport are sedimentation, diffusion, turbulent inertial deposition, inertial deposition in a bend, inertial deposition in a contraction, inertial deposition in an enlargement, electrostatic deposition, thermophoresis, diffusiophoresis, interception and coagulation (Hinds, 1998; Willeke and Baron, 2005) and are described using the "transport efficiency" (Sect. 2.3). Both sampling and transport efficiency are combined to yield an "overall efficiency" for the system (Sect. 2.1).

In the course of writing the Particle Loss Calculator, it was necessary to select formulas best suited to the program from a large variety available in the literature. In order to do so, all available formulas were collated and grouped according to the quantity calculated. Equations for calculating like quantities were compared with one another. Where different formulas resulted in different results, the equation delivering the mean result was chosen and those giving extreme results omitted. Another criterion for the applicability of a formula is its range of validity. A wide range of validity of the implemented relationships is preferable in order to cover the maximum range of particle sizes and conditions in arbitrary tubing systems. Finally, if there were two formulas with similar results and application ranges, we implemented the simpler of the two in order to reduce the probability of programming errors and to minimize computing needs.

Almost all relevant particle loss mechanisms are described well in the literature and implemented in the software. Only the particle loss due to developing eddies in an enlargement was omitted due to unsatisfactory publications and irreproducible calculations. Other loss mechanisms are neglected in the software because their effects are several orders of magnitude smaller than all other contributing terms under normal sampling conditions (see Sect. 2.3.7). Based on these considerations, the following sampling and particle loss mechanisms are included in Particle Loss Calculator:

- Non-isoaxial sampling (Sect. 2.2)

- Non-isokinetic sampling (Sect. 2.2)

- Diffusion (Sect. 2.3.1)

- Sedimentation (Sect. 2.3.2)

- Turbulent inertial deposition (Sect. 2.3.3)

- Inertial deposition in a bend (Sect. 2.3.4)

- Inertial deposition in a contraction (Sect. 2.3.5)

The formulas implemented in the PLC only cover sampling effects through constant-diameter sampling probes. This software cannot be applied to other types of inlet geometries such as shrouded or diffusion inlets.

The equations in the following description were obtained either empirically through experimentation or derived theoretically. Regardless of origin, each equation is only applicable for a limited range of physical conditions whose details can be found under the respective 
subsection for that equation and are also listed in Supplement 1 (see http://www.atmos-meas-tech.net/2/479/2009/ amt-2-479-2009-supplement.zip). An exceeding of the range of validity in the calculation will be marked in the output graph.

If no declaration is given for the unit of a quantity, SIunits are used. A complete list of the parameters as well as two figures showing all angles used in the relationships can be found in Supplement 1. The equations implemented in the Particle Loss Calculator for particle transport represent only a small selection of what is available in the literature. In Sect. 3, the criteria upon which equations were selected are described. For reference, a complete list of the consulted publications can be found in Supplement 2 (see http://www.atmos-meas-tech.net/2/479/2009/ amt-2-479-2009-supplement.zip).

\subsection{Overall efficiency $\eta_{\text {inlet }}$}

In general, the efficiency of a tube is defined as the ratio of the number concentration of particles behind the tube and the number concentration of particles in front of it. The quality of a complete aerosol sampling system is described by the overall inlet efficiency $\eta_{\text {inlet }}$, which is a function of the aerodynamic particle diameter $d_{a}$. The aerodynamic particle diameter can be approximated by

$d_{a}=d_{\text {phys }}\left(\frac{\rho_{p}}{\rho_{0}}\right)^{1 / 2}$

for a wide range of applications (Willeke and Baron, 2005). If the density of the particles $\rho_{p}$ is unknown and set to the standard density $\rho_{0}$ of $1 \mathrm{~g} \mathrm{~cm}^{-3}$, the aerodynamic diameter $d_{a}$ equals the physical diameter $d_{\text {phys }}$ of the particles.

Willeke and Baron (2005) give the overall efficiency as the product of the sampling efficiency $\eta_{\text {sampling }}$ and the transport efficiency $\eta_{\text {transport }}$ :

$\eta_{\text {inlet }}\left(d_{a}\right)=\eta_{\text {sampling }}\left(d_{a}\right) \eta_{\text {transport }}\left(d_{a}\right)$

The sampling efficiency $\eta_{\text {sampling }}$ is the product of the aspiration efficiency $\eta_{\text {asp }}$ and the transmission efficiency $\eta_{\text {trans }}$ of the sampling probe. The transport efficiency $\eta_{\text {transport }}$ is the product of the transport efficiencies for each mechanism operative in each tube section ( $\eta_{\text {tube section,mechanism }}$ ) of the inlet system. In the following sections the individual elements of this overall efficiency are explained in detail.

\subsection{Sampling efficiency $\eta_{\text {sampling }}$}

The sampling efficiency $\eta_{\text {sampling }}$ describes the fraction of aerosol particles that enter the sampling probe from the air surrounding it and successfully reach the transport tubing. This quantity is a composite of the aspiration efficiency $\eta_{\text {asp }}$, the transmission efficiency through the sampling probe due to gravitation $\eta_{\text {trans,grav }}$ and the transmission efficiency due to inertia $\eta_{\text {trans,inert }}$, respectively as a function of the aerodynamic particle diameter $d_{a}$ (Willeke and Baron, 2005):

$\eta_{\text {sampling }}\left(d_{a}\right)=\eta_{\text {asp }}\left(d_{a}\right) \eta_{\text {trans, grav }}\left(d_{a}\right) \eta_{\text {trans,inert }}\left(d_{a}\right)$

In ideal situations the sampling is isoaxial and isokinetic. Isoaxial means that the sampling probe faces straight into the surrounding air motion (wind direction) with no inclination (in general assumed as horizontal). The aspiration angle, $\theta_{S}$, is then $0^{\circ}$. During non-isoaxial sampling, large particles cannot follow the curved streamlines leading into the sampling probe and, as a consequence, miss it (see Fig. 1).

Isokinetic sampling is related to the velocity ratio $R$ relating the local wind speed $U_{0}$ to the flow velocity in the sampling probe $U$ (Willeke and Baron, 2005):

$R=\frac{U_{0}}{U}$

If the surrounding air velocity is higher than the flow velocity in the probe $\left(R>1, U_{0}>U\right)$, sampling is said to be subisokinetic while the opposite $\left(R<1, U_{0}<U\right)$ is termed superisokinetic. In the case of sub-isokinetic sampling, large particles are enriched (in the case of isoaxial sampling) and for super-isokinetic sampling, large particles are depleted (see Fig. 1). In addition three sampling situations are typically distinguished (Willeke and Baron, 2005):

- Sampling in calm air $\left(U_{0}<0.5 \mathrm{~m} \mathrm{~s}^{-1}\right)$

- Sampling in slow-moving air $\left(0.5 \mathrm{~m} \mathrm{~s}^{-1} \leq U_{0} \leq 1.5 \mathrm{~m} \mathrm{~s}^{-1}\right)$

- Sampling in moving air $\left(U_{0}>1.5 \mathrm{~m} \mathrm{~s}^{-1}\right)$

Although there are no commonly agreed upon guidelines for these sampling regimes in the literature, the above criteria were adopted for the calculations in the Particle Loss Calculator. Here sampling in moving air does not include such extreme conditions as occurring during high-speed aircraft sampling. The higher the wind speed the higher the flow velocity inside the inlet tube has to be to obtain isokinetic sampling conditions. We recommend to use the PLC up to wind speeds $U_{0}$ of about $30 \mathrm{~m} \mathrm{~s}^{-1}$. This velocity is a recommendation, there are no commonly agreed upon guidelines for sampling in moving air conditions.

Wiener et al. (1988) show that the influence of ambient air turbulence on the sampling efficiency is negligible. The following relationships can therefore be used for laminar, transitional and turbulent flow conditions surrounding the sampling inlet.

\subsubsection{Aspiration efficiency $\eta_{\text {asp }}$}

The aspiration efficiency $\eta_{\text {asp }}$ is the ratio of the number concentration of particles that enter the sampling probe cross section to the number concentration of particles in the environmental air. Belyaev and Levin $(1972,1974)$ give the 


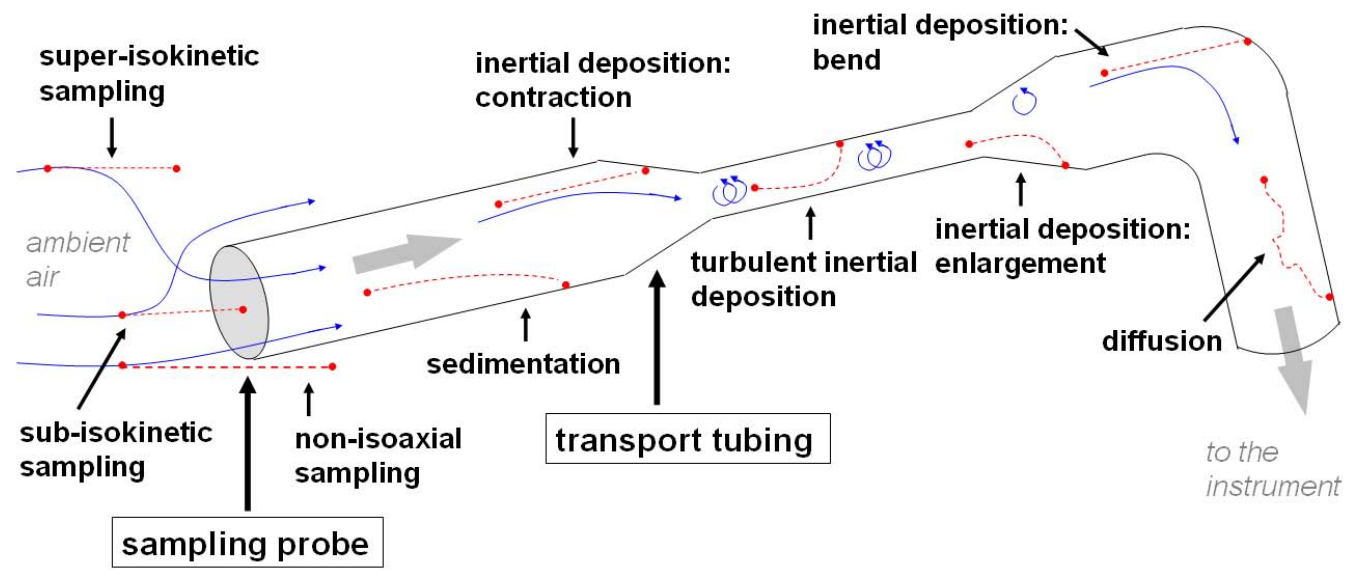

Fig. 1. Mechanisms occurring during aerosol sampling and transport in a sampling probe and a transport tube.

following relationship for the aspiration efficiency in moving air under isoaxial sampling conditions based on a combination of theoretical considerations and experimental data obtained by flash illumination photography:

$\eta_{\text {asp }}\left(d_{a}\right)=1+\left(\frac{U_{0}}{U}-1\right)\left(1-\frac{1}{1+k S t k}\right)$

where $S t k=\left(d_{a}^{2} \rho_{p} C_{C} U_{0}\right) /(18 \mu d)$ is the Stokes Number of the sampling probe (Willeke and Baron, 2005), $\rho_{p}$ is the particle density, $C_{C}=1+K n(1.142+0.558 \exp (-0.999 / K n))$ is the Cunningham slip correction factor (for oil droplets and solid particles) (Allen and Raabe, 1985), $K n=2 \lambda / d_{a}$ is the Knudsen Number, $\lambda$ is the gas molecular mean free path, $\mu$ is the dynamic viscosity of the air (flow medium), $d$ is the inner diameter (ID) of the sampling probe and $k=2+0.617\left(U / U_{0}\right)$. The range of validity for this formula depends on the Stokes Number and the velocity ratio $R$ : $0.05 \leq S t k \leq 2.03$ (Stevens, 1986) and $0.17 \leq U_{0} / U \leq 5.6$.

Under non-isoaxial sampling conditions, the equations of Belyaev and Levin $(1972,1974)$ are no longer valid. For an aspiration angle $\theta_{S}$ from $>0^{\circ}$ to $60^{\circ}$ Durham and Lundgren (1980) give the following equation for the aspiration efficiency based on experiments:

$$
\begin{aligned}
\eta_{\text {asp }}\left(d_{a}\right)= & 1+\left(\frac{U_{0}}{U} \cos \left(\theta_{S}\right)-1\right) . \\
& \frac{1-\left(1+\left(2+0.617\left(U / U_{0}\right)\right) S t k^{\prime}\right)^{-1}}{1-\left(1+2.617 S t k^{\prime}\right)} . \\
& \left(1-\left(1+0.55 S t k^{\prime} \exp \left(0.25 S t k^{\prime}\right)\right)^{-1}\right)
\end{aligned}
$$

where $S t k^{\prime}=S t k \exp \left(0.022 \theta_{S}\right)$. This equation is valid in the ranges $0.02 \leq S t k \leq 4$ and $0.5 \leq U_{0} / U \leq 2$ and was obtained through analysis of several aspiration models and experimental data.
For aspiration angles from $61^{\circ}$ to $90^{\circ}$ Hangal and Willeke (1990a) give:

$\eta_{\text {asp }}\left(d_{a}\right)=1+\left(\frac{U_{0}}{U} \cos \left(\theta_{S}\right)-1\right)\left(3 S t k^{\sqrt{U_{0} / U}}\right)$

for $0.02 \leq S t k \leq 0.2,0.5 \leq U_{0} / U \leq 2$.

If sampling in calm air, gravitational effects are no longer negligible and the terminal settling velocity $V_{t s}$ of the aerosol particles becomes important. The terminal settling velocity is defined in the Stokes Regime (Particle Reynolds Number $\left.R e_{p}<0.1\right)$ as $V_{t s}=\left(\rho_{p} d_{a}^{2} g C_{C}\right) /(18 \mu \chi)$ with $g$ the acceleration of gravity and $\chi$ the dynamic shape factor of the particles (Willeke and Baron, 2005). The terminal settling velocity is the velocity at which the drag force balances the gravitational force (Willeke and Baron, 2005). Grinshpun et al. $(1993,1994)$ compare a theoretically derived expression for aspiration efficiency to experimental data:

$\eta_{\text {asp,calm air }}\left(d_{a}\right)=\frac{V_{t s}}{U} \cos (\varphi)+\exp \left(-\frac{4 S t k^{1+\sqrt{\frac{V_{t s}}{U}}}}{1+2 S t k}\right)$

where $\varphi$ is the angle corresponding to the vertical $\left(\varphi=0^{\circ}\right.$ : vertical sampling). The equation is valid in the ranges $0^{\circ} \leq \varphi \leq 90^{\circ}, 10^{-3} \leq V_{t s} / U \leq 1$ and $10^{-3} \leq S t k \leq 100$.

If the surrounding air is in the slow motion regime, Grinshpun et al. $(1993,1994)$ give another relationship that combines the aspiration efficiency of moving air with that of calm air:

$\eta_{\text {asp , overall }}\left(d_{a}\right)=\eta_{\text {asp }}(1+\delta)^{1 / 2} f_{\text {moving }}+\eta_{\text {asp ,calm air }} f_{\text {calm }}$

where

$\delta=\left(V_{t s} / U_{0}\right)\left(V_{t s} / U_{0}+2 \cos \left(\theta_{S}+\varphi\right)\right)$.

$f_{\text {moving }}=\exp \left(-V_{t s} / U_{0}\right)$ and $f_{\text {calm }}=1-\exp \left(-V_{t s} / U_{0}\right)$ are the interpolation weighting factors and $V_{t s}=V_{0}-U_{0} . V_{0}$ is the initial velocity of the particles. The equation is valid in the ranges $-90^{\circ} \leq \varphi \leq 90^{\circ}$ and $-90^{\circ} \leq \theta_{S} \leq 90^{\circ}$. 
These formulations are only valid for thin-walled sampling probes for which the particle loss due to particle bounce on the edge of the probe can be neglected. A sampling probe can be regarded as thin-walled when the ratio of its outer to inner diameter is less than 1.1 (Belyaev and Levin, 1972). Although different relationships are available for blunt samplers, the use of blunt samplers should be avoided for most applications.

\subsubsection{Transmission efficiency of the sampling probe $\eta_{\text {trans }}$}

The transmission efficiency $\eta_{\text {trans }}$ is the ratio of the aerosol particle number concentration behind the sampling probe to the particle number concentration in front of the sampling probe. The fractional particle loss is one minus the transmission efficiency. The particle loss in the sampling probe due to gravitational and inertial forces is expressed by the transmission efficiencies $\eta_{\text {trans, grav }}$ and $\eta_{\text {trans,inert }}$ :

$\eta_{\text {trans }}\left(d_{a}\right)=\eta_{\text {trans,grav }}\left(d_{a}\right) \eta_{\text {trans,inert }}\left(d_{a}\right)$

The transmission efficiency of sampling probes for gravitational effects is described by Okazaki et al. (1987a,b). However, Yamano and Brockmann (1989) point out that this formula underestimates the transmission efficiency due to several invalid assumptions. Gravitational effects are better taken into account in the calculation of transport losses and therefore it is not necessary to consider them as part of the sampling efficiency as well. However, inertial effects directly related to the sampling process are important for the sampling efficiency. Liu et al. (1989) give an expression for the transmission efficiency based on numerical simulations of particle trajectories (isoaxial sampling):

$\eta_{\text {trans,inert }}\left(d_{a}\right)=\frac{1+\left(U_{0} / U-1\right) /\left(1+2.66 S t k^{-2 / 3}\right)}{1+\left(U_{0} / U-1\right) /(1+0.418 / S t k)}$

for $1 \leq U_{0} / U \leq 10$ and $0.01 \leq S t k \leq 100$.

Coefficients are derived from the publications of Belyaev and Levin $(1972,1974)$ and are the result of fits to experimental data. Hangal and Willeke (1990a,b) assume that the formation of eddies in the sampling probe enhance the deposition of particles for super-isokinetic sampling $(R<1)$. They give a theoretically derived relationship for the transmission efficiency in this range:

$\eta_{\text {trans,inert }}\left(d_{a}\right)=\exp \left(-75 I_{v}^{2}\right)$

where

$I_{v}=0.09\left(\operatorname{Stk}\left(U-U_{0}\right) / U_{0}\right)^{0.3}$.

These equations are valid in the ranges $0.02 \leq S t k \leq 4$ and $0.25 \leq U_{0} / U \leq 1$.

For non-isoaxial sampling, they give an extended equation:

$\eta_{\text {trans,inert }}\left(d_{a}\right)=\exp \left(-75\left(I_{v}+I_{w}\right)^{2}\right)$ where

$I_{v}=0.09\left(\text { Stk } \cos \left(\theta_{S}\right)\left(U-U_{0}\right) / U_{0}\right)^{0.3}$

for $0.25 \leq U_{0} / U \leq 1$ and $I_{v}=0$ otherwise,

$I_{w} \Downarrow=S t k \sqrt{U_{0} / U} \sin \left(\theta_{S}-\alpha\right) \sin \left(\left(\theta_{S}-\alpha\right) / 2\right)$

the direct impaction loss parameter for downward sampling (sampling probe faces upward),

$I_{w} \Uparrow=S t k \sqrt{U_{0} / U} \sin \left(\theta_{S}+\alpha\right) \sin \left(\left(\theta_{S}+\alpha\right) / 2\right)$

the direct impaction loss parameter for upward sampling (sampling probe faces downward) and

$\alpha=12\left(\left(1-\theta_{S} / 90\right)-\exp \left(-\theta_{S}\right)\right)$.

These equations are valid in the ranges $0.02 \leq S t k \leq 4$, $0.25 \leq U_{0} / U \leq 4$ and $0^{\circ}<\theta_{S} \leq 90^{\circ}$.

\subsection{Transport losses $\eta_{\text {transport }}$}

The fraction of aerosol particles lost during aerosol transport through the inlet system is expressed using the transport efficiency, $\eta_{\text {transport }}$. This quantity is the ratio of the number concentration of particles leaving a tube to the number concentration of particles entering the tube. The transport loss is one minus the transport efficiency. Willeke and Baron (2005) give the following expression for the overall transport efficiency through an inlet setup:

$\eta_{\text {transport }}\left(d_{a}\right)=\prod_{\text {tube sections }}\left(\prod_{\text {mechanisms }} \eta_{\text {tube section,mechanism }}\left(d_{a}\right)\right)$,

i.e. the overall transport efficiency through an inlet system is the product of the transport efficiencies for all tube sections of the transport tubing and for all particle loss mechanisms. There are different relationships in the literature for the particle loss occurring in the transport tubing depending on the flow conditions in the tube based on the Reynolds Flow Number $R e$ (Willeke and Baron, 2005). Some equations are only valid for the laminar $(R e<2000)$ or turbulent flow regime ( $R e>4000)$ while other formulas cover the whole range of flow conditions. For the transition regime, $2000<R e<4000$, no formula is available. To carry out calculations also in this flow regime the PLC offers the option to extend the laminar equations through the transition regime. These estimated particle losses have a lower precision than those in the laminar and turbulent regime. However, they are still useful for a basic estimation of occurring losses.

\subsubsection{Diffusion $\eta_{\text {diff }}$}

For particles smaller than $100 \mathrm{~nm}$, Brownian motion creates a net flux of particles from areas with high concentrations towards areas with low concentrations. The walls of a tube are a sink for small particles creating an area of low concentration near them. Because of this, diffusion always generates a 
net transport of particles to the walls where they deposit. For laminar flow conditions in a tube, Willeke and Baron (2005) give an equation for the transport efficiency associated with diffusion:

$\eta_{\text {diff }}\left(d_{a}\right)=\exp (-\xi S h)$

where $S h$ is the Sherwood Number, $\xi=\pi D L / Q, D$ is the particle diffusion coefficient, $L$ is the tube length and $Q$ is the flow rate.

For the Sherwood Number a formula by Holman (1972) can be used:

$S h=3.66+\frac{0.0668 \frac{d}{L} \operatorname{Re} S c}{1+0.04\left(\frac{d}{L} \operatorname{Re} S c\right)^{2 / 3}}=3.66+\frac{0.2672}{\xi+0.10079 \xi^{1 / 3}}$

where $R e=\rho_{f} U d / \mu$ is the Reynolds Flow Number, $\rho_{f}$ is the density of the air (the flow medium), $U$ is the flow velocity in the tube, $d$ is the inner tube diameter and $S c=\mu /\left(\rho_{f} D\right)$ is the Schmidt Number.

If the flow in a tube is turbulent, the formula from Willeke and Baron (2005) (Eq. 21) is used with the experimentally obtained Sherwood Number given by Friedlander and Johnstone (1957):

$S h=0.0118 R e^{7 / 8} S c^{1 / 3}$

\subsubsection{Sedimentation $\eta_{\text {grav }}$}

For particles having a diameter larger than about $0.5 \mu \mathrm{m}$, gravitational forces cause particle loss. These particles settle out due to their weight inside the tube, depositing on the lowermost surface as dictated by the acceleration of gravity. For laminar flow in a horizontal tube Fuchs (1964) and Thomas (1958) give the following relation, which is based on a parabolic flow profile:

$$
\begin{gathered}
\eta_{\text {grav }}\left(d_{a}\right)=1-\frac{2}{\pi}\left(2 \epsilon \sqrt{1-\epsilon^{2 / 3}}-\epsilon^{1 / 3} .\right. \\
\left.\sqrt{1-\epsilon^{2 / 3}}+\arcsin \left(\epsilon^{1 / 3}\right)\right)
\end{gathered}
$$

where $\epsilon=3 / 4 Z$ and $Z=L V_{t s} /(d U)$. $Z$ is the so called gravitational deposition parameter and $V_{t s}$ is the terminal settling velocity of the particles.

If the tube is inclined with respect to horizontal by an angle of inclination of $\theta_{i}$, Heyder and Gebhart (1977) used experiments to derive a modified equation for the sedimentation loss:

$$
\begin{gathered}
\eta_{\text {grav }}\left(d_{a}\right)=1-\frac{2}{\pi}\left(2 k^{\prime} \sqrt{1-k^{\prime 2 / 3}}-k^{\prime 1 / 3} .\right. \\
\left.\sqrt{1-k^{\prime 2 / 3}}+\arcsin \left(k^{\prime / 3}\right)\right)
\end{gathered}
$$

where $k^{\prime}=\epsilon \cos \left(\theta_{i}\right)$ and the condition $V_{t s} \sin \left(\theta_{i}\right) / U \ll 1$ must be satisfied.
Under turbulent flow conditions the correlations of Schwendiman et al. (1975) have to be used. Here the transport efficiency due to sedimentation loss in a horizontal tube is:

$\eta_{\text {grav }}\left(d_{a}\right)=\exp \left(-\frac{4 Z}{\pi}\right)=\exp \left(-\frac{d L V_{t s}}{Q}\right)$

and for an inclined tube:

$\eta_{\text {grav }}\left(d_{a}\right)=\exp \left(-\frac{4 Z \cos \left(\theta_{i}\right)}{\pi}\right)=\exp \left(-\frac{d L V_{t s} \cos \left(\theta_{i}\right)}{Q}\right)$.

As in the laminar case the condition $V_{t s} \sin \left(\theta_{i}\right) / U \ll 1$ must be fulfilled.

\subsubsection{Turbulent inertial deposition $\eta_{\text {turb inert }}$}

The turbulent inertial deposition is the inertial deposition loss of large particles due to the curved streamlines (eddies) in a turbulent flow. Large particles cannot follow these streamlines due to their high inertia and are deposited on the walls of the tube. Willeke and Baron (2005) give a relation for the transport efficiency associated with this effect:

$\eta_{\text {turb inert }}\left(d_{a}\right)=\exp \left(-\frac{\pi d L V_{t}}{Q}\right)$

where

$V_{t}=\frac{\left(6 \times 10^{-4}\left(0.0395 \text { Stk } R e^{3 / 4}\right)^{2}+2 \times 10^{-8} R e\right) U}{5.03 R e^{1 / 8}}$

is the experimentally determined turbulent inertial deposition velocity. Equation 28 is valid in the turbulent flow regime up to a Reynolds Number of 15600 (Lee and Gieseke, 1994).

\subsubsection{Inertial deposition: bend $\eta_{\text {bend,inert }}$}

In a bend in tubing, the streamlines of the flow change their direction and large particles cannot follow them perfectly due to their inertia. Whether they will be deposited on the walls of the tubing as a result of their inability to follow flow lines depends on particle stopping distance. For laminar flow Pui et al. (1987) give an empirical relation for the transport efficiency associated with this loss mechanism:

$\eta_{\text {bend,inert }}\left(d_{a}\right)=\left(1+\left(\frac{S t k}{0.171}\right)^{0.452 \frac{S t k}{0.171}+2.242}\right)^{-\frac{2}{\pi} \theta_{K r}}$

where $\theta_{K r}$ is the angle of curvature of the bend in degrees.

Pui et al. (1987) also provide an empirically determined relationship for the inertial particle loss in a bend in tubing in turbulent flow:

$\eta_{\text {bend,inert }}\left(d_{a}\right)=\exp \left(-2.823\right.$ Stk $\left.\theta_{K r}\right)$

The effect of the curvature ratio $R_{0}$ on the inertial deposition in a bend is insignificant for $5 \leq R_{0} \leq 30$ (Pui et al., 1987). The curvature ratio $R_{0}$ is defined as the radius of the bend divided by the radius of the tube (Willeke and Baron, 2005). 


\subsubsection{Inertial deposition: contraction $\eta_{\text {cont,inert }}$}

In a contraction in tubing, there is also a change in the direction of the streamlines which larger particles cannot completely follow. As a consequence, particles may deposit on the walls in front of the contraction. Muyshondt et al. (1996b) give a relationship for the transport efficiency obtained through experiments using particle collection on filters both in front of and behind a contraction:

$$
\eta_{\text {cont,inert }}\left(d_{a}\right)=1-\frac{1}{1+\left(\frac{S t k\left(1-\left(\frac{A_{o}}{A_{i}}\right)\right)}{3.14 \exp \left(-0.0185 \theta_{\text {cont }}\right)}\right)^{-1.24}},
$$

which is valid in the ranges $0.001 \leq \operatorname{Stk}\left(1-A_{o} / A_{i}\right) \leq 100$ and $12^{\circ} \leq \theta_{\text {cont }} \leq 90^{\circ}$. For this equation, $\theta_{\text {cont }}$ is the contraction half-angle, $A_{i}$ is the cross-sectional area in front of the contraction, and $A_{o}$ is the cross-sectional area behind the contraction.

\subsubsection{Inertial deposition: enlargement}

In an enlargement in a piece of tubing, eddies form if the angle of enlargement is larger than $8^{\circ}$ (or, in other words, if the half-angle is larger than $4^{\circ}$ ) (Schade and Kunz, 1989). The eddies cause curved streamlines towards the tube walls and potentially causing particle deposition behind the enlargement. As there is no suitable equation describing this effect in the literature, care should be taken when designing an inlet that angles of enlargement be kept small to avoid the development of eddies (Willeke and Baron, 2005). The general advice is to experimentally determine occurring particle losses if it is not possible to avoid an enlargement in an inlet system.

\subsubsection{Effects not considered in the Particle Loss Calculator}

Electrostatic deposition: the loss of charged aerosol particles due to electrostatic deposition is negligible if the sampling lines are grounded and consist of conductive material (e.g. metal). Under these circumstances, no electrical field will exist in the interior of the tube (Faraday cage) and even highly charged aerosol particles will not be electrostatically deposited (Willeke and Baron, 2005). One exception to this is in the case of unipolar charged aerosol particles where mutual particle repulsion will produce a net flux of the particles towards the walls causing deposition. Under most measurement situations, aerosol particles are not unipolar charged and this case can be neglected.

Thermophoresis: if a temperature gradient exists within the tubing, a net flux of aerosol particles develops from hot to cold areas in a tube. This is due to the difference in momentum of the air molecules as a function of temperature. On the hotter side, air molecules transfer more momentum to the particles than on the colder side resulting in particle transport towards the colder side. If the walls are colder than the air inside the tube, aerosol particles get lost to the walls. In the opposite situation particle loss is reduced. Under most ambient aerosol measurement situations the temperature gradient between the tube walls and the aerosol is smaller than $40 \mathrm{~K}$ and the particle loss due thermophoresis is negligible. This has been mathematically confirmed for several air thermal conductivities by the authors.

Diffusiophoresis: the deposition of aerosol particles due to concentration gradients can generally be neglected, if the sampled air is well mixed and the temperature gradient between aerosol and sampling lines is not too extreme. This is important in order to avoid the condensation of gas molecules on the tubing walls, which would produce a concentration gradient. These conditions are given under normal ambient aerosol measurement conditions (Willeke and Baron, 2005).

Interception: interception is the process by which particles travelling on streamlines sufficiently close to a tube wall eventually come into contact with the wall, stick to it, and deposit. This effect is much smaller than other particle loss processes if the dimensions of the particle are much smaller than the dimensions of the tube. In most inlet transport situations this condition is fulfilled and interception can be neglected (Willeke and Baron, 2005).

Coagulation: coagulation is the conglomeration of many smaller aerosol particles into fewer large ones. This process swiftly decreases the small aerosol particle number concentration while more slowly increasing the number concentration of large particles (Willeke and Baron, 2005). The aerosol particle loss due to coagulation can be neglected if particle concentrations are smaller than 100000 particles per $\mathrm{cm}^{3}$ and if the residence time of the aerosol in the sampling lines amounts to only a few seconds. This has been mathematically confirmed by the authors.

Re-entrainment of deposited particles: re-entrainment of particles is a not well-characterized process and should be avoided by cleaning the inlet lines, providing laminar flow conditions, reducing sedimentation of particles and minimization of mechanical shock and vibration to the inlet system (Willeke and Baron, 2005). It is important to consider, that re-entrained particles do not represent the current air mass. Even if the actual losses of large particles are slightly lower due to re-entrainment, we think it is the best way to assume a higher particle loss for large particles and not to account for the re-entrainment of particles.

\section{Basic working principle of the Particle Loss Calculator}

Generally, there are two approaches for calculation of particle losses in an inlet system. One approach involves the use of computational fluid dynamics (CFD) algorithms to numerically simulate the air flow and particle transport through the system. The other is the use of empirical and theoretically derived formulas as described in Sect. 2 for individual tube sections and the calculation of the overall efficiency of the total inlet system using Eq. (2). 
CFD applications use numerical methods to solve complex coupled systems of equations (Navier-Stokes Equations) that describe fluid dynamical problems. Using such methods it is not only possible to calculate the gas flow field through an aerosol inlet system, but also to determine aerosol particle distributions and particle trajectories. CFD calculations are the method of choice for the characterization of aircraft inlets subject to high sampling velocities or other sampling situations subject to similar conditions. The advantages of this approach are, among other things, its wide range of applicability and the detailed representation of flow profiles in tubing. Particle loss can be determined by the calculation of particle trajectories and a detailed insight into the processes occurring in a tube system is possible (CFD Review, 2009).

In spite of the power of this approach, one significant disadvantage of computational fluid dynamics is the complexity inherent in defining necessary input parameters (e.g. the geometry of the calculated object and the calculation grid). Proper use of CFD software is only possible by trained users and is very time consuming to learn. In addition, the complexity of the numerical algorithms used in computation means that calculations themselves consume a great deal of computational power. For these reasons, CFD calculations are not well suited for quick, flexible estimates of particle losses in an inlet system that are routinely encountered when designing measurement systems. Furthermore, Tian and Ahmadi (2006) have shown that CFD calculations of particle losses occurring during turbulent aerosol sampling and transport are often not reliable. Whereas the equations implemented in the PLC are the results of experiments done with turbulent flows, so they can be assumed to be more reliable to correctly describe the influence of turbulent sampling and transport.

The use of empirical and theoretically derived formulas was the method of choice for the Particle Loss Calculator to make calculations for arbitrary inlet systems accessible for those not trained in CFD. This approach has already been applied in the "AeroCalc" collection of Excel spreadsheets (Willeke and Baron, 2005). These spreadsheets contain more than 100 equations, largely detailed in Willeke and Baron (2005) and Hinds (1998), for the calculation of aerosol parameters like the air viscosity, the slip correction factor and the particle relaxation time. Using these spreadsheets, it is also possible to calculate particle losses in aerosol inlet systems by combining appropriate formulas. Kumar et al. (2008) also used this approach, when they compared measurements of ultrafine particle loss to theoretical determinations based on the laminar flow model of Gormley and Kennedy (1949) and the turbulent flow model of Wells and Chamberlain (1967).

While "AeroCalc" is a multifunctional tool for the calculation of a large variety of aerosol parameters, the Particle Loss Calculator is specially designed to streamline the combination of these calculations for efficient estimation of particle losses in arbitrary aerosol inlet systems. The Particle
Loss Calculator was written using the scientific graphing and data analysis environment "IGOR Pro 6.04" (WaveMetrics, 2009). It has a simple and clearly arranged user interface making the collated theoretical and experimental information found in a large selection of literature sources accessible to all users. The results of the Particle Loss Calculator have also been experimentally validated.

\subsection{Particle Loss Calculator (PLC)}

The basic working principle of the Particle Loss Calculator is presented in Fig. 2. As described in Sect. 2, we separated the calculation of the total inlet sampling efficiency into two parts. The first part is the calculation of the sampling efficiency of the sampling probe. This quantity is composed of the aspiration and the transmission efficiency (Eq. 3) and accounts only for effects associated with the sampling of aerosol particles from ambient air into the tubing. The second part of the calculation concerns transport efficiency of aerosols through tubing to the measurement instrument. For calculation of transport efficiency, the inlet system is separated into simple tube sections and the individual transport efficiencies for each section are calculated for each loss mechanism (Eq. 20). The total inlet efficiency is the combination of the sampling efficiency of the sampling probe and the transport efficiency through the transport lines (Eq. 2). All calculations are performed for each particle size in a user selectable size range and in user selectable size steps to achieve a size-resolved quantity. The Particle Loss Calculator can be set to calculate the efficiency of either one of these processes or the combination of both (overall efficiency/inlet efficiency).

The user interface of the resulting software Particle Loss Calculator is presented in Fig. 3. Six boxes logically organize the input parameters that must be entered to perform the calculation. The "Parameters of the Sampling"-box is used to define the variables for the computation of the inlet sampling efficiency. To perform such a calculation, the "Account for Sampling Effects"-check box must be activated. Otherwise, when the "Action"-button is pressed, a warning text appears. Parameters used for the calculation of the inlet sampling efficiency are the "Sampling Orientation", the "Aspiration Angle", the "Orifice Diameter", the "Flow Rate" and the "Wind Velocity". The sampling orientation of the inlet can be set as horizontal, upward (the aerosol is drawn from high to low into the tube) or downward (the aerosol is drawn from low to high into the tube). The aspiration angle (in degrees) gives the deviation of the sampling probe direction from the wind direction (regardless of whether the derivation is in horizontal or vertical direction). The orifice diameter in $\mathrm{mm}$ is the inner diameter of the tube opening, at the point where the aerosol enters the tubing. The flow rate in $1 \mathrm{~min}^{-1}$ is that measured in the first tube section immediately downstream of the orifice, and the wind velocity in $\mathrm{m} \mathrm{s}^{-1}$ is the speed of the surrounding air in relation to the sampling probe. 


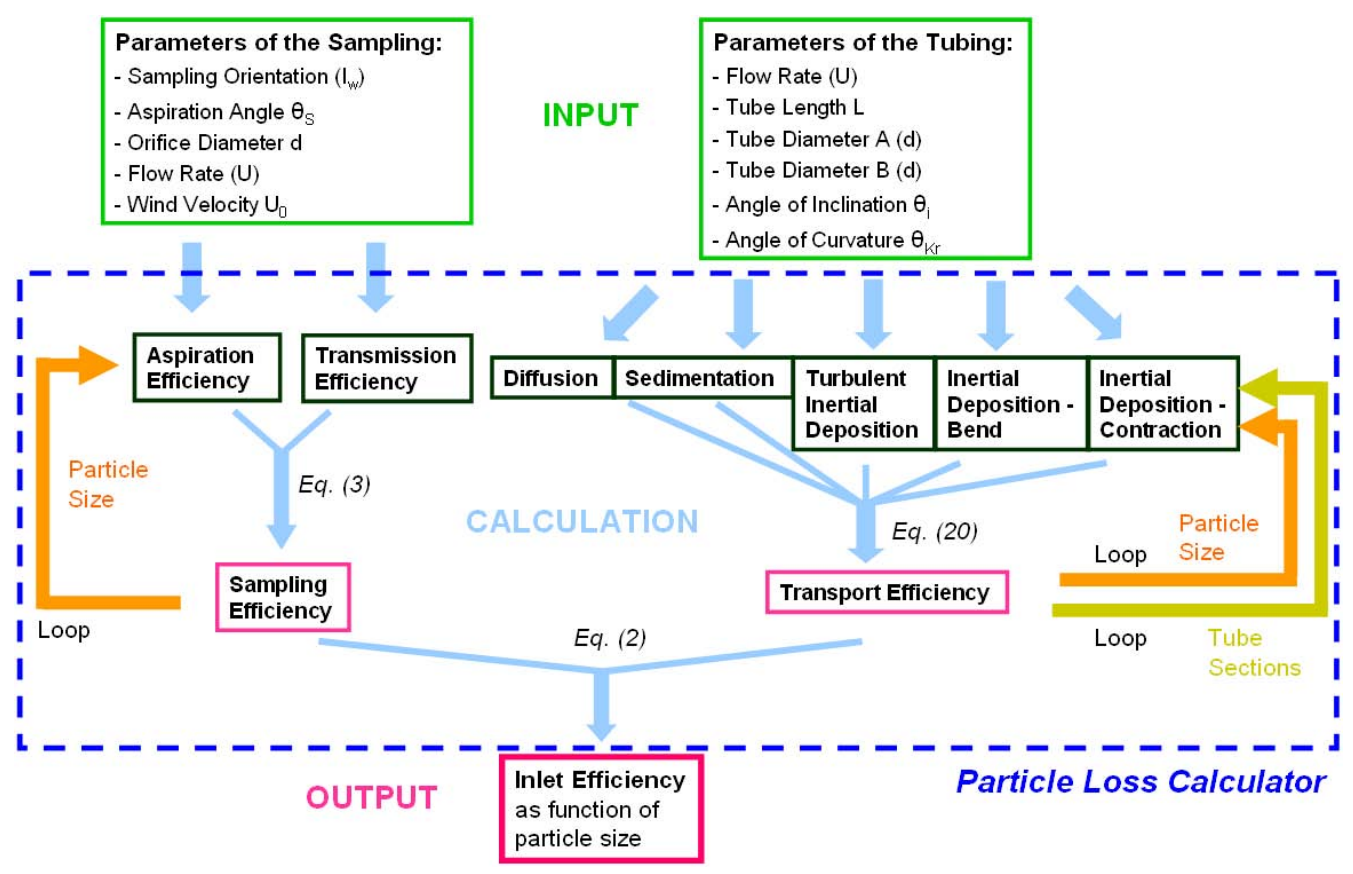

Fig. 2. Basic working principle of the Particle Loss Calculator. In the green input boxes the variables in brackets are calculated from the respective listed parameters, the variables without brackets are the listed parameters itself.

It is important to note that the orifice diameter and the flow rate required to calculate inlet sampling effects are also used as parameters for calculating transport efficiency in the first tube section. If these two parameters of the sampling probe are different from the values set for the first tube section, an error message is displayed.

The "Parameters of the Tubing"-box contains necessary input for calculation of the transport efficiency. First, the user sets the number of tubing sections to be used for the calculation (maximum 100). For this software, a tubing section is defined according to constant parameters, e.g. a straight tube or a bend of a certain angle. Any time one of the dimensions of the tubing of an inlet changes, a new tubing section must be started. After selecting the number of sections, the user can choose to load or edit the parameters by clicking the corresponding button. These buttons call a table containing the parameters of the tube sections (see Fig. 4). The first line of the table contains the parameters of the first tubing section for the calculation of the transport efficiency, the second line those of the second tubing section and so on. The following parameters have to be set for each tube section: "Flow Rate", "Tube Length", "Tube Diameter A", "Tube Diameter B", "Angle of Inclination" and "Angle of Curvature". The unit of the flow rate is $1 \mathrm{~min}^{-1}$, the tube length is in $\mathrm{m}$, the diameters are in $\mathrm{mm}$ and the angles are in degrees. The "Tube Diameter A" is related to the inner tube diameter at the beginning (the first part of the tube encountered by air as it flows through the tube) of a tube section. "Tube Diameter B" is the

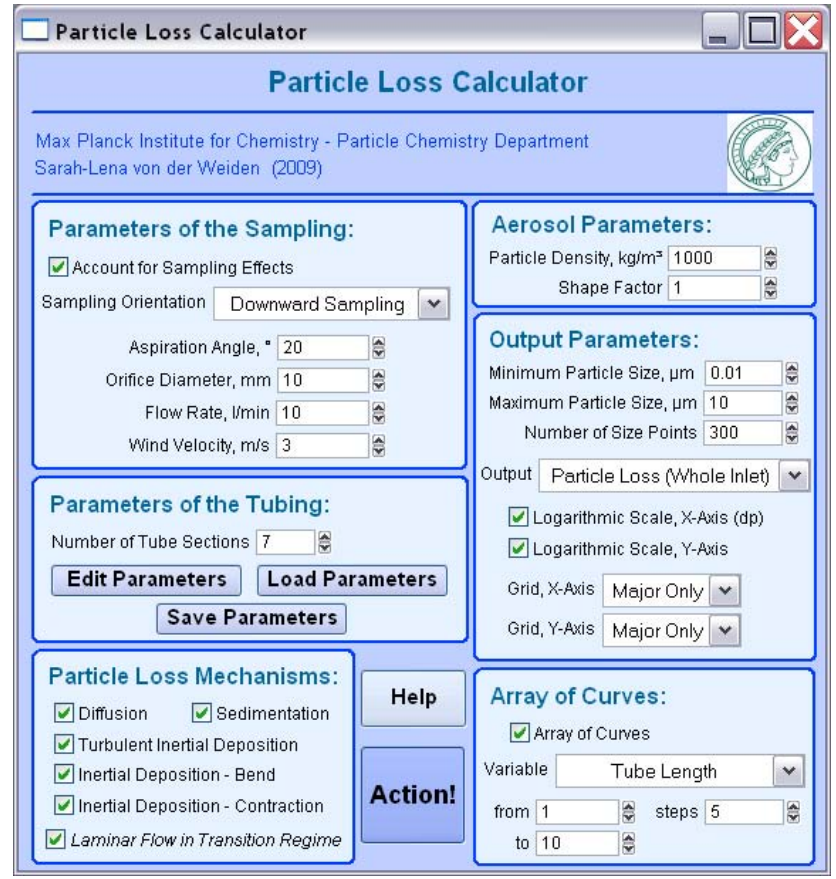

Fig. 3. User interface of the Particle Loss Calculator. 
inner diameter of the end (the last part of the tube encountered by air as it flows through the tube) of a tube section. In the case of an enlargement or a contraction, the values for "A" and "B" will be different. For a straight tube section both diameters "A" and "B" are the same. The angle of inclination is defined with respect to the horizontal plane. The angle of enlargement or contraction is calculated depending on the "Tube Diameter A", the "Tube Diameter B" and the "Tube Length". As discussed previously, particle loss due to developing eddies in an enlargement with an angle larger than $4^{\circ}$ are not considered in the calculation. If this angle is too large, a message is displayed explaining that the calculated particle loss is underestimated. For later use of a tube system the parameters of the tubing can be saved with the corresponding button.

The "Particle Loss Mechanisms"-box allows the user to choose which of the implemented mechanisms are included in the calculation. These mechanisms are diffusion, sedimentation, turbulent inertial deposition, inertial deposition in a bend and inertial deposition in a contraction. The user can include any number or combination of these mechanisms in the calculation allowing either general estimates of transport losses or investigation of the contribution of individual mechanisms to the overall process.

To enable calculations for the transition regime for which no relationships exist, the formulas for the laminar flow regime can be extended to non-laminar conditions by checking the "Laminar Flow in Transition Regime"-box. A warning text will appear in the output graph pointing out that these calculations are outside of the valid range for the relationships employed. If this option is not chosen and the flow conditions in one or more tube sections are in the transition regime, no calculation of the particle loss is possible and an appropriate warning will appear.

The "Aerosol Parameters"-box is used to define the particle density and the shape factor for the aerosol to be sampled. The default value of the particle density is $1000 \mathrm{~kg} \mathrm{~m}^{-3}$, corresponding to the density of water. The shape factor is 1 for spherical particles and larger than 1 for other shapes (Seinfeld and Pandis, 2006). If the characteristics of the sampled aerosol particles are known, these parameters can be changed appropriately.

The "Output Parameters"-box contains variables that determine the appearance of the output window displaying calculated results. As mentioned above, the user can choose to calculate either individual loss processes or the combination of all effects. In this window, the user selects which results to display as well as the particle size range and number of steps within this range that should be displayed ("Number of Size Points"). The chosen quantity, either percent efficiency or loss, is plotted on the $y$-axis versus the particle size in $\mu \mathrm{m}$ on the x-axis.

The "Array of Curves"-box is used to set the parameters required for the calculation of an array of curves with variation of one of the sampling or tubing parameters. This feature can be used to determine optimum parameters of an inlet system during the design phase. One of several variables affecting the sampling or the transportation processes can be varied in an user-selectable number of steps. The user sets the start ("from") and the end ("to") value of the respective variable. For such calculations, the aspiration angle, the orifice diameter, the flow rate and the wind velocity can be varied. These quantities are marked with an "(S)" in the "Variable" menu. If one of them is chosen, the calculated quantity (Output Parameters, "Output" menu) has to be the sampling efficiency or the sampling loss. Otherwise a warning appears. For the transport efficiency all parameters in the parameter table and additionally the angle of contraction can be selected for an array of curves. The angle of enlargement cannot be varied, because the effects of an enlargement are not implemented in the calculations. The determination of an array of curves is possible only for a single tube section (or inlet sampling conditions) and the variables for this section have to be set in the first row of the parameter table.

To support the user in applying the Particle Loss Calculator a detailed help text ("Help"-button) explains all functions and parameters of this software. Additionally, the software prints information in the status line concerning individual elements when the cursor is over the each of the six areas in the panel. The calculation of sampling and transport losses starts by pressing the "Action"-button at the bottom of the panel. After a short time either the output window appears displaying the chosen quantity as a function of particle diameter or one of the mentioned notifications points out that an input parameter is wrong.

The output graph can contain a blue dashed line, a red solid line or both to present the chosen quantity. If a blue dashed line (in the legend shown as " $X$ ") appears, one or more of the formulas used are out of their validity range. The result of the calculation is then an approximation. A red line (in the legend shown as "N") indicates that all formulas are within their stated validity range. In practice, the result of a calculation is often still useful even if a formula is used outside its limits of validity. This is particularly true of the equations applying to inlet sampling effects which seem to have a narrower stated validity than is actually allowable.

\section{Validation measurements}

To verify the functionality and practicability of the Particle Loss Calculator, we compared experimentally determined particle losses in several simple test tube systems to the results of the Particle Loss Calculator. For the calculations using the Particle Loss Calculator, all particle loss mechanisms were selected and therefore tested. 


\begin{tabular}{|c|c|c|c|c|c|c|c|}
\hline 圈 $\mathrm{T}$ & ableo: No, flowr at & ,tubelength,... & & & & $\square \square 8$ & (3) \\
\hline & R8 & 0 & & & & $\nabla$ & \\
\hline No & Flow Rate, I/min & Tube Length, $m$ & Tube Diameter A, mm & Tube Diameter B, mm & Angle of Inclination, ${ }^{\circ}$ & Angle of Curvature, ${ }^{\circ}$ & \\
\hline 1 & 4 & 0.05 & 10 & 10 & 0 & 0 & ^ \\
\hline 2 & 4 & 0.1 & 10 & 20 & 45 & 0 & 国 \\
\hline 3 & 4 & 0.1 & 20 & 5 & 0 & 0 & \\
\hline 4 & 1.2 & 0.083 & 5 & 10 & 0 & 0 & \\
\hline 5 & 1.2 & 0.05 & 10 & 10 & 90 & 45 & \\
\hline 6 & 1.2 & 0.033 & 10 & 3 & 90 & 0 & \\
\hline 7 & 3 & 1.2 & 3 & 3 & 0 & 90 & $v$ \\
\hline$<$ & & & & & & $\rightarrow$ & \\
\hline
\end{tabular}

Fig. 4. Table containing the parameters of the tubing.

\subsection{Experimental setup}

Experimentally determined particle losses were calculated with the following equation:

particle loss $(\%)=\left(1-\frac{\text { number conc. of particles at tube exit }}{\text { number conc. of particles at tube entrance }}\right) \cdot 100 \%$

Two identical Condensation Particle Counters (CPCs, TSI, model 3007) and Optical Particle Counters (OPCs, Grimm, model 1.109) were used for the detection of particles at tubing entrances and exits in the size range from about $10 \mathrm{~nm}$ to $350 \mathrm{~nm}$ and $300 \mathrm{~nm}$ to $32 \mu \mathrm{m}$, respectively. To reliably determine particle losses, the instruments were tested to determine that they respond identically when measuring the same aerosol.

The CPCs measure the number of aerosol particles per $\mathrm{cm}^{3}$ independent of size. To obtain size-resolved measurements of particle loss using a CPC, monodisperse aerosol particles having a variety of sizes must be generated and tested separately. For CPCs experiments, aerosol particles were generated using an atomizer spraying aqueous ammonium sulfate solution. The emerging droplets were dried in an aerosol dryer filled with silica gel and the remaining particles were led into a Differential Mobility Analyzer (DMA, TSI, model 3081) which was used to select particles of specific sizes from the polydisperse aerosol. A comparison of the CPCs sampling from the same aerosol showed a small difference in instrument response independent of particle size. For all subsequent experiments, a correction factor of 1.0094 was used to scale the response of one of the CPCs such that it exactly matched the response of the second.

The OPCs measure the aerosol particle concentration (particles per liter) in 31 different size channels from particles larger than $0.25 \mu \mathrm{m}$ ranging to particles larger than $32 \mu \mathrm{m}$. These two instruments were used to sample ambient air in a variety of locations. Over the course of the measurements, there was large size dependent discrepancy between signals (up to $40 \%$ ) for the two OPCs although they were sampling the same aerosol. Using this data, we derived a size dependent correction factor with which to scale the results of one instrument to match the other (see Table 1 correction factor for outdoor validation measurements). Correction factors for both OPCs and CPCs were confirmed at regular time intervals over the course of validation measurements to verify their stability.

For small particles $(<300 \mathrm{~nm})$ the effects of diffusion and to some extent sedimentation are important while for large particles $(>0.5 \mu \mathrm{m})$ those of inertial deposition (for example, in a bend) and sedimentation dominate the overall loss. We experimentally determined the particle losses of small particles for five different test tubes with different lengths, curvatures, and diameters. The particle losses of large particles were determined for three different tubes, two designed mainly for impaction losses (large total angle of curvature with short length) and one mainly for sedimentation losses (large horizontal extension). The flow conditions in all experiments were in the laminar flow regime.

\subsection{Results of the validation measurements}

For the validation experiments for the diffusional loss calculations of small particles, we used stainless steel $1 / 4$ inch (ID=4.57 mm) and $1 / 2$ inch $(\mathrm{ID}=10.00 \mathrm{~mm}$ ) tubes of several lengths at low flow velocities. The $1 / 4$ inch tubes had lengths of $20.80 \mathrm{~m}, 10 \mathrm{~m}$ and $3 \mathrm{~m}$ and were coiled in several turns (up to 10). The experimentally determined particle losses show similar trends to the calculated losses. However, in the size range from about $20 \mathrm{~nm}$ to $200 \mathrm{~nm}$ the measured particle losses are higher than the calculated losses. Measurements made with varying numbers of turns ( 0 up to 18 coils) of the tubes show that the difference between measured and calculated losses depends on the angle of curvature. With an increased number of turns, particle losses increased. Particle loss due to inertial effects (e.g. in curves) is expected to be negligible for small particles in a laminar flow in the range tested. Nevertheless, these results show that geometry has a strong influence on the aerosol particle losses. The structure of the flow seems to depend not only on the Reynolds Number, but also on the geometry of the tube, at least the extreme we tested. As such an effect is not implemented in the calculation of particle losses, we do not recommend its use for calculations involving extreme geometries. We advise to keep inlet designs simple (avoidance of extreme curvature) to avoid possible excessive particle losses. 
Table 1. Correction factor applied to one of the OPCs during the outdoor validation measurements.

\begin{tabular}{|c|c|c|c|c|c|c|c|c|}
\hline \multicolumn{9}{|l|}{ Particle Size $(\mu \mathrm{m})$} \\
\hline & 0.265 & 0.290 & 0.325 & 0.375 & 0.425 & 0.475 & 0.54 & 0.615 \\
\hline & 0.675 & 0.750 & 0.900 & 1.150 & 1.450 & 1.800 & 2.250 & 2.750 \\
\hline & 3.250 & 4.500 & 5.750 & 7.000 & 8.000 & 9.250 & 11.250 & 13.750 \\
\hline & 16.250 & 18.750 & 22.500 & 27.500 & 31.000 & & & \\
\hline \multicolumn{9}{|l|}{ Correction Factor } \\
\hline & 0.841 & 0.998 & 1.015 & 0.968 & 0.840 & 0.609 & 1.028 & 0.870 \\
\hline & 0.920 & 1.101 & 0.816 & 1.125 & 0.930 & 0.903 & 0.963 & 0.925 \\
\hline & 0.881 & 0.912 & 0.833 & 0.985 & 0.868 & 1 & 1 & 1 \\
\hline & 1 & 1 & 1 & 1 & 1 & & & \\
\hline
\end{tabular}

In order to further validate the Particle Loss Calculator, we used tubes with less extreme geometries. In Fig. 5 the calculated and measured particle losses for two straight $1 / 2$ inch tubes with lengths of $21 \mathrm{~m}$ and $6.85 \mathrm{~m}$ are shown. The aerosol particle loss in percent is plotted on the y-axis versus the aerosol particle size (mobility diameter) in $\mathrm{nm}$ on the $\mathrm{x}$-axis. The points are the results of the measurements and the lines are the calculated particle losses for the tube geometries used in the measurements. The error bars are the standard deviation of a series of five measurements. The experimentally determined particle losses are consistent with the calculated losses. This software tool can therefore be assumed to function well in this size range and for simple geometries where diffusion is the dominating particle loss process. Below a particle size of about $20 \mathrm{~nm}$, calculated results cannot be validated as the DMA could not generate reliable monodisperse aerosol below this size.

To validate the calculation of sedimentation and inertial deposition for larger particles, three tubes with different geometries were tested. To obtain better counting statistics, some measurements were carried out near a busy street, where larger concentrations of large aerosol particles were available than in laboratory. Test tube configurations used for this measurement (1/4 inch-tube, total angle of curvature: $720^{\circ}$, length: $0.35 \mathrm{~m}$ ) are presented in Fig. 6 along with the results of the tests. The line is the calculated particle loss for the given tube geometry and the dots are the results of the measurements. Errors in the measurement are derived from counting statistics related to the total number of particles measured in each size channel. The measured particle losses agree very well with the calculated losses up to a particle size of about $7 \mu \mathrm{m}$. The larger variation in the measured particle losses between $200 \mathrm{~nm}$ and $2 \mu \mathrm{m}$ may be related to unidentified external factors affecting determination of the OPC correction factor.

The results for two other tubes, one designed for inertial deposition (1/4 inch-tube, total angle of curvature: $1080^{\circ}$, length: $0.68 \mathrm{~m}$ ) and the other designed for sedimentation losses (1/2 inch-tube, length: $0.66 \mathrm{~m}$, no curvature), are not

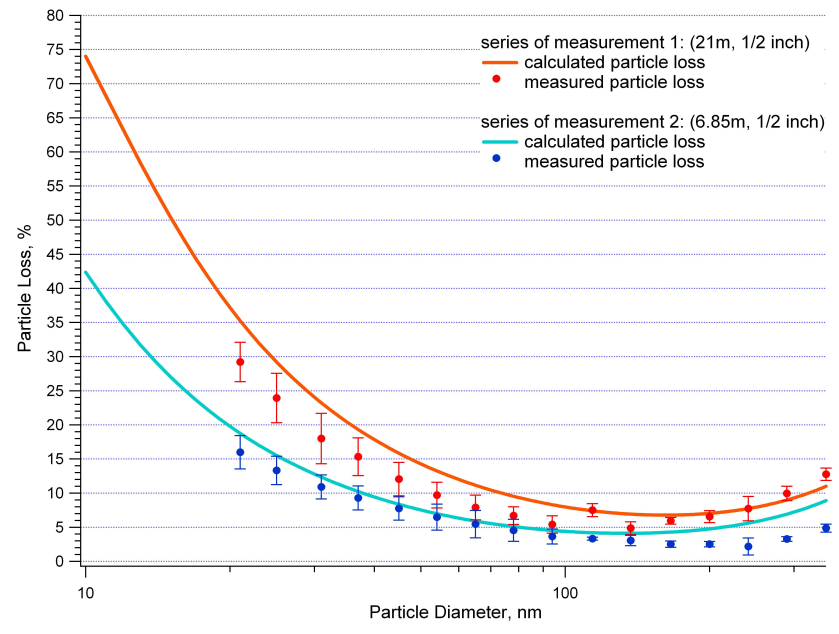

Fig. 5. Measured and calculated particle losses in two 1/2 inch tubes without curves and a length of $21 \mathrm{~m}$ (series of measurement 1 , red dots and line) and $6.85 \mathrm{~m}$ (series of measurement 2 , blue dots and line).

shown here. However, results are comparable to those shown in Fig. 6 with very good agreement between experimentally determined and calculated particle losses. The Particle Loss Calculator appears to function well for this size range where sedimentation and inertial deposition are the dominating particle loss mechanisms.

\section{Applications of the Particle Loss Calculator}

In this section we present three applications demonstrating the use and utility of the Particle Loss Calculator. Figure 7 depicts a virtual non-isoaxial and non-isokinetic sampling system in conjunction with transport tubing designed for high particle losses during transport. The green numbers demarcate individual tube sections used for the calculation. All other necessary parameters for the calculation with the 


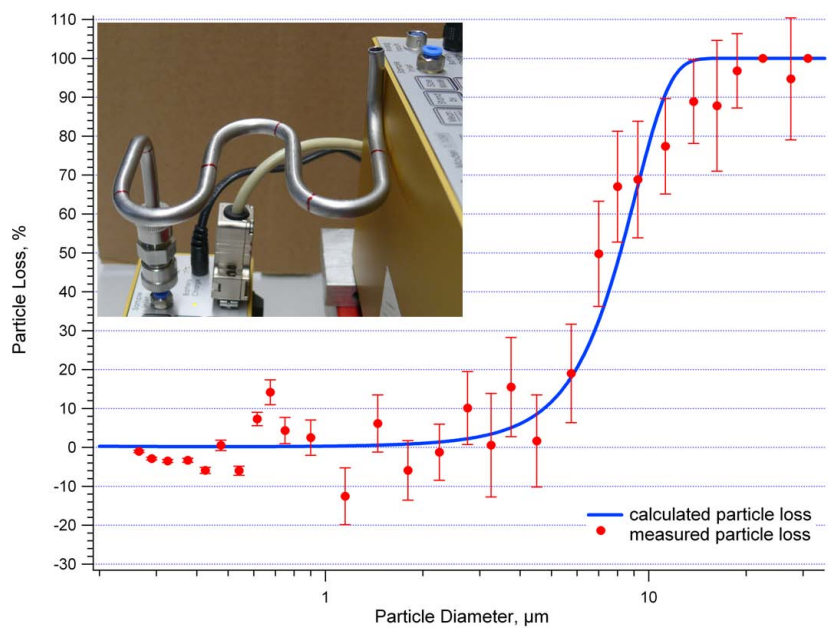

Fig. 6. Measured (red dots) and calculated (blue line) particle losses in a 1/4 inch tube designed for impaction losses in bends.

Particle Loss Calculator can also be taken from this figure. This inlet system was purposely designed to demonstrate the potential impact of poor inlet system design on aerosol sampling. In the lower panel of Fig. 7, the size dependent particle loss occurring in the virtual tube system for three different flow conditions is shown. The particle loss in percent is plotted versus the particle diameter in $\mu \mathrm{m}$. The red curve (case 1) is the result using a flow rate of $101 \mathrm{~min}^{-1}$ where there is a laminar flow profile in all tube sections. Particle losses are below $10 \%$ for a large size range and even drop under a value of $1 \%$ for particles between $100 \mathrm{~nm}$ and $600 \mathrm{~nm}$. Up to a particle size of a few $\mu \mathrm{m}$ the characteristics of the inlet system are acceptable for laminar flow conditions and the results of an instrument measuring in this size range would likely be not negatively influenced.

The black curve (case 2) is the particle loss occurring in the inlet system if a flow rate of $401 \mathrm{~min}^{-1}$ is used. In tube sections 1 to 5 laminar flow conditions prevail, while in the small diameter tube of section 6 , the flow is turbulent. The resulting particle losses are clearly higher than in case 1 . For all particle diameters the losses are at least 5\%. Only for particles larger than $1 \mu \mathrm{m}$ are the losses slightly smaller due to a shorter residence time in the inlet system reducing sedimentation losses. In general, the sampling conditions are worse in case 2 than in case 1 with non-negligible particle losses evident for all sizes.

Case 3 (green curve) depicts the sampling conditions producing that largest artifacts. Here, a flow rate of $1501 \mathrm{~min}^{-1}$ causes turbulent flow conditions in all tube sections. The resulting particle losses are at least $40 \%$ for all particle sizes and particles larger than $3 \mu \mathrm{m}$ are not able to reach the measurement instrument at all. Such sampling conditions should be avoided as meaningful measurements are impossible under these circumstances.
This example shows the utility of the Particle Loss Calculator for assessing the characteristics of an inlet system and for adjusting sampling conditions to minimize losses. The Particle Loss Calculator could further be used to correct results from existing systems to account for size dependent loss processes or to estimate measurement errors.

As mentioned previously, the Particle Loss Calculator was developed in order to optimize the aerosol inlet system for the mobile laboratory MoLa of the Max Planck Institute for Chemistry in Mainz, the goal being to minimizing particle losses across all size fractions to whatever extent possible and provide correction factors should losses be non-negligible for a given size fraction or instrument. Several boundary conditions existed for this task including vehicle layout, already existing inlet ports and tubes, the characteristics of the measurement instruments and the different measurement conditions during stationary and mobile measurements. Inlet efficiencies and particle losses had to be calculated numerous times to best optimize this system including variations in tube routing, tube diameter, flow velocity, arrangement of valves, inlet lines for each measurement instrument, sampling probes for several driving speeds and the design of curved tube sections. Optimum particle loss in this case did not result in lowest losses everywhere, but rather a combination of low loss for the largest possible range of particle sizes within the measured size range of the individual instruments on board such that losses, when they did occur, had minimal impact.

In Fig. 8 the calculated particle losses for three measurement instruments installed in MoLa (AMS, ELPI, TEOM) operated with the roof inlet are shown. The particle losses in percent are plotted versus the particle diameter in $\mu \mathrm{m}$ and the particle losses are shown across the measurement size range of the respective instrument. For the AMS the calculated losses are below $2 \%$ over a wide size range, for the ELPI below $10 \%$ and for the TEOM below $1 \%$. The particle losses are negligible for these three instruments when sampling through the MoLa roof inlet. The inlet losses for the other instruments and the other two inlet systems of MoLa are of the same magnitude in as wide a size range as those shown in Fig. 8.

Yet another example of the use of the Particle Loss Calculator can be found in the publication of Sagharfifar et al. (2009). Here this software was applied to determine the particle losses in the inlet system and the humidification chamber of a modified condensation particle counter. The results of these calculations were used to estimate the overall error of the instrument.

\section{Summary}

Accurate aerosol measurements taken under changing or drastically variable sampling conditions place high demands on inlet systems used to sample aerosols. Optimization and 

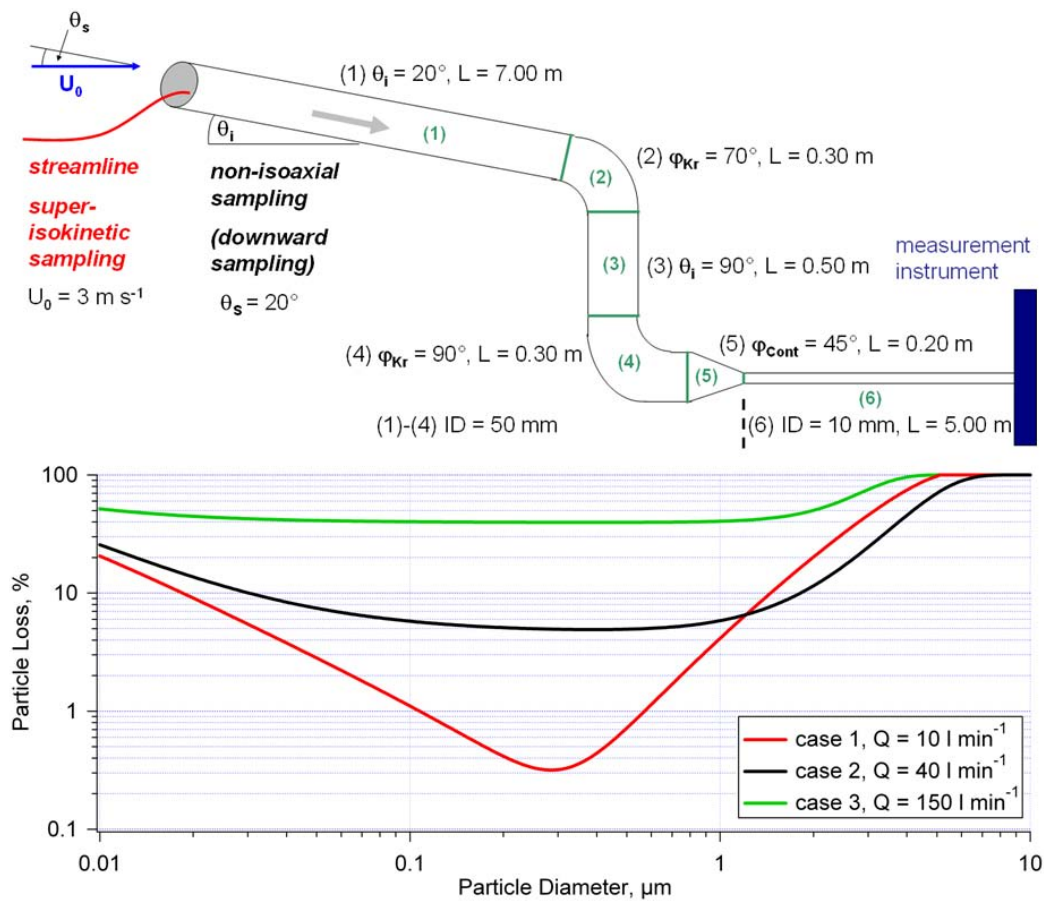

Fig. 7. Application example of the Particle Loss Calculator (virtual tube system not drawn to scale).

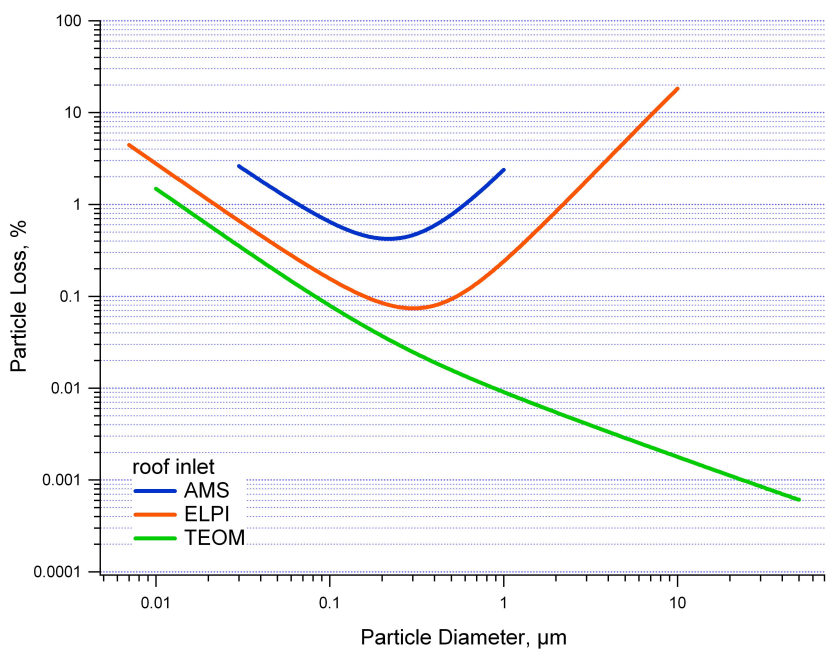

Fig. 8. Calculated particle losses for three measurement instruments installed in MoLa (AMS, ELPI, TEOM) operated with the roof inlet.

characterization of inlet systems is necessary to obtain representative aerosol sampling, to preserve the main characteristics of the ambient aerosol, and ensure scientifically significant results.

We developed a new Particle Loss Calculator (PLC) program, based on both empirically and theoretically derived relations that can be used for the assessment of the perfor- mance of existing aerosol inlet systems or development of new ones. The Particle Loss Calculator helps to quickly determine aerosol sampling efficiencies and particle transport losses for arbitrary tubing systems as a function of particle size. In developing this software, based on stepwise calculations for individual tube sections, we reviewed the processes influencing the sampling and transport of aerosol particles currently described in the literature and implemented those processes strongly influencing particle loss under common sampling situations. Where multiple parameterizations for a loss process exist, the optimal parameterization was chosen for implementation. This software was further validated by comparison with experimentally determined particle losses observed in several simple test systems. As long as tube geometries are not too extreme, calculations using the Particle Loss Calculator program agree well with experiment.

Three examples demonstrate potential applications for the Particle Loss Calculator. Calculations involving a virtual inlet system show the potentially deleterious effects of using inlet systems with large and poorly characterized losses. In addition, two real-world examples of inlet design are given. One describes the utility of Particle Loss Calculator in designing the inlet for the new MoLa mobile laboratory in Mainz and the second describes its use in characterizing the inlet of a modified condensation particle counter.

The Particle Loss Calculator is a software under continuous development and suggestions for its improvement are welcome. 
Acknowledgements. We thank Sören Zorn for helpful discussions, Thomas Böttger for support with the validation measurements and Thomas Custer for proofreading. Furthermore, we acknowledge the University of Mainz and the Max Planck Institute for Chemistry for funding of this work.

The service charges for this open access publication have been covered by the Max Planck Society.

Edited by: D. Toohey

\section{References}

Allen, M. and Raabe, O.: Slip Correction Measurements of Spherical Solid Aerosol-Particles in an Improved Millikan Apparatus, Aerosol Sci. Tech., 4, 269-286, 1985.

Appel, B. R., Povard. V., and Kothny, E. L.: Loss of Nitric Acid Within Inlet Devices Intended to Exclude Coarse Particles During Atmospheric Sampling, Atmos. Environ., 22(11), 25352540, 1988.

Belyaev, S. and Levin, L.: Investigation of Aerosol Aspiration by Photographing Particle Tracks Under Flash Illumination, J. Aerosol Sci., 3, 127-140, 1972.

Belyaev, S. and Levin, L.: Techniques for Collection of Representative Aerosol Samples, J. Aerosol Sci., 5, 325-338, 1974.

Brunekreef, B. and Holgate, S. T.: Air Pollution and Health, The Lancet, 360, 1233-1242, 2002.

Bukowieki, N., Dommen, J., Prevot, A., Richter, R., Weingartner, E., and Baltensperger, U.: A Mobile Pollutant Measurement Laboratory - Measuring Gas Phase and Aerosol Ambient Concentrations With High Spatial and Temporal Resolution, Atmos. Environ., 36, 5569-5579, 2002.

CFD Review, http://cfdreview.com, access: 20 August 2009.

Davies, C.: Aerosol Science, Academic Press, 1966.

Drewnick, F., Hings, S. S., DeCarlo, P., Jayne, J. T., Gonin, M., Fuhrer, K., Weimer, S., Jimenez, J. L., Demerjian, K. L., Borrmann, S., and Worsnop, D. R.: A New Time-of-Flight Aerosol Mass Spectrometer (TOF-AMS) - Instrument Description and First Field Deployment, Aerosol Sci. Tech., 39, 637-658, 2005.

Durham, M. and Lundgren, D.: Evaluation of Aerosol Aspiration Efficiency as a Function of Stokes Number, Velocity Ratio and Nozzle Angle, J. Aerosol Sci., 11, 179-188, 1980.

Friedlander, S. and Johnstone, H.: Deposition of Suspended Particles from Turbulent Gas Streams, Ind. Eng. Chem., 49, 11511156, 1957.

Fuchs, N.: The Mechanics of Aerosols, Pergamon, Oxford, 1964.

Fuchs, N.: Sampling of Aerosols, Atmos. Environ., 9, 697-707, 1975.

Gard, E., Mayer, J. E., Morrical, B. D., Dienes, T., Fergenson, D. P., and Prather, K. A.: Real-Time Analysis of Individual Atmospheric Aerosol Particles: Design and Performance of a Portable ATOFMS, Anal. Chem., 69, 20, 4083-4091, 1997.

Gormley, P. G. and Kennedy, M.: Diffusion From a Stream Following Through a Cylindrical Tube, Proceedings of Royal Irish Academy, 52, 163-169, 1949.

Grinshpun, S., Willeke, K., and Kalatoor, S.: A General Equation for Aerosol Aspiration by Thin-Walled Sampling Probes in Calm and Moving Air, Atmos. Environ. A - Gen., 27, 1459-1470, 1993.
Grinshpun, S., Willeke, K., and Kataloor, S.: Corrigendum: A General Equation for Aerosol Aspiration by Thin-Walled Sampling Probes in Calm and Moving Air (27A, 1459-1470, 1993), Atmos. Environ., 28, p. 375, 1994.

Hangal, S. and Willeke, K.: Aspiration Efficiency - Unified Model for All Forward Sampling Angles, Environ. Sci. Technol., 24, 688-691, 1990a.

Hangal, S. and Willeke, K.: Overall Efficiency of Tubular Inlets Sampling at 0-90 Degrees From Horizontal Aerosol Flows, Atmos. Environ. A - Gen., 24, 2379-2386, 1990 b.

Heyder, J. and Gebhart, J.: Gravitational Deposition of Particles From Laminar Aerosol Flow Through Inclined Circular Tubes, J. Aerosol Sci., 8, 289-295, 1977.

Hinds, W.: Aerosol Technology: Properties, Behavior, and Measurement of Airborne Particles, Wiley-Interscience, New York, 1998.

Holman, J.P.: Heat Transfer, McGrawHill, New York, 1972.

Huebert, B. J., Lee, G., and Warren, W. L.: Airborne Aerosol Inlet Passing Efficiency Measurement, J. Geophys. Res., 95(D10), 16369-16381, 1990.

Intergovernmental Panel on Climate Change (IPCC), World Meteorological Organisation and United Nations Environment Programme, United Nations, 2007.

Kittelson, D., Johnson, J., Watts, W., Wei, Q., Drayton, M., and Paulsen, D.: Diesel Aerosol Sampling in the Atmosphere, SAE Technical Papers Series, 2000.

Kolb, C., Herndon, S., McManus, B., Shorter, J., Zahniser, M., Nelson, D., Jayne, J., Canagaratna, M., and Worsnop, D.: Mobile Laboratory with Rapid Response Instruments for Real-Time Measurements of Urban and Regional Trace Gas and Particulate Distributions and Emission Source Characteristics, Environ. Sci. Technol., 38, 5694-5703, 2004.

Kumar, P., Fennell, P., Symonds, J., and Britter, R.: Treatment of Losses of Ultrafine Aerosol Particles in Long Sampling Tubes During Ambient Measurements, Atmos. Environ., 42, 88198826, 2008.

Lee, K. and Gieseke, J.: Deposition of Particles in Turbulent Pipe Flows, J. Aerosol Sci., 25, 699-709, 1994.

Levin, L.: The Intake of Aerosol Samples, Izv. Akad. Nauk SSSR Ser. Geofiz., 7, 914-925, 1957.

Liu, B., Zhang, Z., and Kuehn, T.: A Numerical Study of Inertial Errors in Anisokinetic Sampling, J. Aerosol Sci., 20, 367-380, 1989.

McMurry, P. H.: A Review of Atmospheric Aerosol Measurements, Atmos. Environ., 34, 1959-1999, 2000.

Muyshondt, A., McFarland, A., and Anand, N.: Deposition of Aerosol Particles in Contraction Fittings, Aerosol Sci. Tech., 24, 205-216, 1996b.

Okazaki, K., Wiener, R., and Willeke, K.: The Combined Effect of Aspiration and Transmission on Aerosol Sampling Accuracy for Horizontal Isoaxial Sampling, Atmos. Environ., 21, 1181-1185, 1987a.

Okazaki, K., Wiener, R., and Willeke, K.: Isoaxial Aerosol Sampling - Nondimensional Representation of Overall Sampling Efficiency, Environ. Sci. Technol., 21, 178-182, 1987b.

Orsini, D. A., Ma, Y. L., Sullivan, A., Sierau, B., Baumann, K., and Weber, R. J.: Refinements to the Particle-into-Liquid Sampler (PILS) for Ground and Airborne Measurements of Water Soluble Aerosol Composition, Atmos. Environ., 37(9-10), 1243-1259, 
2003.

Pirjola, L., Parviainen, H., Hussein, T., Valli, A., Hameri, K., Aaalto, P., Virtanen, A., Keskinen, J., Pakkanen, T. A., Makela, T., and Hillamo, R. E.: "Sniffer" - A Novel Tool for Chasing Vehicles and Measuring Traffic Pollutants, Atmos. Environ., 38, 3625-3635, 2004.

Pui, D., Romaynovas, F., and Liu, B.: Experimental Study of Particle Deposition in Bends of Circular Cross Section, Aerosol Sci. Technol., 7, 301-315, 1987.

Sagharfifar, H., Kuerten, A., Curtius, J., von der Weiden, S.-L., Hassanzadeh, I., and Borrmann, S.: Characterization of a Modified Expansion Condensation Particle Counter for Detection of Nanometer-Sized Particles, Aerosol Sci. Technol., 43(8), 767780, 2009.

Schade, H. and Kunz, E.: Strömungslehre, de Gruyter Lehrbuch, Berlin, New York, 1989.

Schwendiman, L., Stegen, G., and Glissmeyer, J.: Report BNWLSA-5138, 1975.

Seinfeld, J. and Pandis, S.: Atmospheric Chemistry and Physics: From Air Pollution to Climate Change, Wiley \& Sons, 2006.

Stevens, D.: Review of Aspiration Coefficients of Thin-Walled Sampling Nozzles, J. Aerosol Sci., 17, 729-743, 1986.

Thomas, J.: Gravity Settling of Particles in a Horizontal Tube, JAPCA J. Air Waste Ma., 8, 32-34, 1958.

Tian, L. and Ahmadi, G.: Particle Deposition in Turbulent Duct Flows - Comparison of Different Model Predictions, J. Aerosol Sci., 38(4), 377-397, 2006.
Vincent, J.: Aerosol Sampling, Science and Practice, WileyInterscience, New York, 1989.

WaveMetrics: IGOR Pro 6, http://wavemetrics.com, access: $20 \mathrm{Au}-$ gust 2009.

Weber, R. J., Orsini, D., Daun, Y., Lee, Y. N., Klotz, P. J., and Brechtel, F.: A Particle-Into-Liquid Collector for Rapid Measurements of Aerosol Bulk Chemical Composition, Aerosol Sci. Tech., 35(3), 718-727, 2001.

Wells, A. C. and Chamberlain, A. C.: Transport of Small Particles to Vertical Surfaces, Brit. J. Appl. Phys., 18, 1793-1799, 1967.

Wiener, R., Okazaki, K., and Willeke, K.: Influence of Turbulence on Aerosol Sampling Efficiency, Atmos. Environ., 22, 917-928, 1988.

Willeke, K. and Baron, P.: Aerosol Measurement: Principles, Techniques, and Applications, Van Nostrand Reinhold, New York, 2005.

Winklmayr, W., Reischl, G. P., Lindner, A. O., and Berner, A.: A New Electromobility Spectrometer for the Measurement of Aerosol Size Distributions in the Size Range From 1 to 1000 NM, J. Aerosol Sci., 22(3), 289-296, 1991.

Yamano, N. and Brockmann, J.: Aerosol Sampling and Transport Efficiency Calculation (ASTEC) and Application to Surtsey/DCH Aerosol Sampling System, NUREG/CR-525. SAND88-1447, Albuquerque, NM: Sandia National, 1989. 